7-1-1997

\title{
Monte Carlo Study of the Square-Lattice Annealed Ising Model on Percolating Clusters
}

\author{
P. D. Scholten \\ Miron Kaufman \\ Cleveland State University, m.kaufman@csuohio.edu
}

Follow this and additional works at: https://engagedscholarship.csuohio.edu/sciphysics_facpub

Part of the Physics Commons

How does access to this work benefit you? Let us know!

\section{Publisher's Statement}

Copyright 1997 American Physical Society. Available on publisher's site at http://link.aps.org/ doi/10.1103/PhysRevB.56.59.

\section{Original Citation}

Scholten, P. D. and Miron Kaufman. "Monte Carlo Study of the Square-Lattice Annealed Ising Model on Percolating Clusters." Physical Review B 56 (1997): 59-62.

\section{Repository Citation}

Scholten, P. D. and Kaufman, Miron, "Monte Carlo Study of the Square-Lattice Annealed Ising Model on Percolating Clusters" (1997). Physics Faculty Publications. 49.

https://engagedscholarship.csuohio.edu/sciphysics_facpub/49

This Article is brought to you for free and open access by the Physics Department at EngagedScholarship@CSU. It has been accepted for inclusion in Physics Faculty Publications by an authorized administrator of EngagedScholarship@CSU. For more information, please contact library.es@csuohio.edu. 


\title{
Monte Carlo study of the square-lattice annealed Ising model on percolating clusters
}

\author{
P. D. Scholten \\ Department of Physics, Miami University, Oxford, Ohio 45056 \\ Miron Kaufman \\ Department of Physics, Cleveland State University, Cleveland, Ohio 44115
}

(Received 24 January 1997)

\begin{abstract}
Simulations of an Ising $q$-state Potts model which is equivalent to the Ising model on annealed percolation clusters are used to determine the phase diagram of the model in two dimensions. Three topologically different phase diagrams are obtained: (i) for $q=2$, there are two critical Ising lines meeting at $T=0$ at the four-state Potts critical point; (ii) for $2<q \leqslant 4$, the Ising critical line meets the $q$-state critical line and a line of first-order transitions at a bicritical point; (iii) for $q>4$, the Ising critical line intersects a line of first-order transitions at a critical end point. [S0163-1829(97)06725-8]
\end{abstract}

\section{INTRODUCTION}

The annealed Ising model on percolation clusters was shown ${ }^{1}$ to show interesting phase diagrams from a positionspace renormalization-group technique. In particular, that study has suggested the existence of novel multicritical points. Though physical systems such as fluids in porous media and amorphous magnets are better described by quenched randomness, ${ }^{2}$ it is useful to have the annealed model as a proxy for the more realistic but also less tractable quenched model.

In this paper we present a Monte Carlo simulation of the model on a square lattice to validate the renormalizationgroup predictions. ${ }^{1}$ The simulations were carried out on an Ising- $q$-state Potts model which is equivalent ${ }^{1}$ to the Ising model on percolation clusters. There are three thermodynamic fields: the fugacity $q$ controlling the number of clusters, the probability $p$ for the presence of bonds, and the temperature or the inverse Ising coupling. Note that the percolation process ${ }^{3}$ considered here allows for correlations between bonds. The random-bond percolation holds for $q=1$. We have studied the phase diagrams for $q=2,3,4,6$, and 10. Though the gross features of the renormalization-group phase diagram, e.g., the three phases of nonpercolating, percolating paramagnetic, and percolating ferromagnetic, are confirmed by the Monte Carlo method, there are important differences between the results of the two methods. For $q$ $=3$ and 4 , the Monte Carlo method predicts the occurrence of a bicritical point ${ }^{4}$ at the intersection of two continuous transitions lines (one Ising like and the other Potts like) with a line of first-order transitions. For higher $q$ (we looked at $q=6$ and 10) we find a critical end point ${ }^{5,6}$ at the intersection of the Ising transition line with two lines of first-order transitions. We have also simulated the model for $q=2$. In this case we seem to confirm the position-space renormalization-group ${ }^{1}$ prediction: two Ising critical lines meeting at $T=0$ at the four-state Potts multicritical point.

The remainder of the paper is organized as follows. In Sec. II we present the model and review some exact results which are used to verify the numerical simulations. Section III contains our Monte Carlo simulations. The conclusions are included in Sec. IV.

\section{THEORY}

At each site of the square lattice, there is an Ising spin $s_{i}= \pm 1$ and a Potts spin $\sigma_{i}=1,2, \ldots, q$. The Hamiltonian is

$$
-H / k_{B} T=\sum_{\langle i, j\rangle}\left[J \delta\left(\sigma_{i}, \sigma_{j}\right)+L \delta\left(\sigma_{i}, \sigma_{j}\right) S_{i} S_{j}\right],
$$

where $\delta$ is the Kronecker delta and $\langle i, j\rangle$ means nearest neighbors. (Note that this Hamiltonian differs from the dilute Potts model of Nienhuis et al. ${ }^{7}$ ) This model was shown ${ }^{1}$ to be equivalent to the Ising model on annealed percolation clusters:

$$
Z=\sum_{\text {conf }} q^{c} w^{B} Z_{I}^{\mathrm{conf}}
$$

where $w=p /(1-p), p$ is the probability for a bond to be present, $C$ is number of clusters, and $B$ is number of bonds. $Z_{I}^{\text {conf }}$ is the Ising partition function for the particular percolation configuration:

$$
Z_{I}^{\mathrm{conf}}=\operatorname{Tr}_{\left(S_{i}\right)} \exp \left[K \sum_{\langle i, j\rangle}\left(S_{i} S_{j}-1\right)\right],
$$

where $\langle i, j\rangle$ means a pair of sites connected by a bond. The parameters $J$ and $L$ of Eq. (1) are related to $w$ and $K$ of Eqs. (2) and (3) as follows:

$$
\begin{aligned}
& J=\frac{1}{2} \ln \left[(1+w)\left(1+w e^{-2 K}\right)\right], \\
& L=\frac{1}{2} \ln \left[(1+w) /\left(1+w e^{-2 K}\right)\right] .
\end{aligned}
$$

Some exact results can be deduced from the Ising-Potts model. If $K=0$ from Eq. (4), we get $L=0$ : (i.e., this is the $q$-state Potts model with critical coupling, $J_{c}=\ln (1+\sqrt{q})$, which corresponds to $p_{c}=\sqrt{q} /(1+\sqrt{q})$.

If $K=\infty$, from Eq. (4) we get $J=L$. The Hamiltonian becomes

$$
-H / k_{B} T=\sum_{\langle i, j\rangle}\left[2 J \delta\left(\sigma_{i}, \sigma_{j}\right) \delta\left(S_{i}, S_{j}\right)\right] .
$$


This is the $2 q$-state Potts model with critical coupling $J_{c}$ $=\frac{1}{2} \ln (1+\sqrt{2 q})$, which corresponds to $p_{c}=\sqrt{2 q} /(1+\sqrt{2 q})$.

For $p=1$ the Hamiltonian in Eq. (1) reduces to the Ising model

$$
-H / k_{B} T=\sum_{\langle i, j\rangle} K S_{i}, S_{j},
$$

apart from an additive constant. The critical coupling is $K_{c}$ $=\frac{1}{2} \ln (1+\sqrt{2})$.

Finally, the order of the Potts transitions in two dimensions is known. ${ }^{8}$ It is continuous if $q \leqslant 4$ and it is discontinuous if $q>4$.

\section{MONTE CARLO SIMULATIONS}

Monte Carlo (MC) simulations based on the Hamiltonian of Eq. (1) were carried out for systems with $q=2,3,4,6$, and 10 on square lattices of sizes $\boldsymbol{L}=14,20,30$, and 40 . Each site was considered to be in one of $2 q$ possible states, and the Metropolis algorithm was used to determine changes in the states. Periodic boundary conditions were used for all lattices, and each MC simulation consisted of $5 \times 10^{6}$ lattice passes after the first $5 \times 10^{4}$ passes had been discarded to help ensure equilibration. For the statistical analysis a complete two-dimensional probability distribution of the Ising and Potts energies, $P\left(E_{I}, E_{P}\right)$, was collected for each simulation. From this the system energy and heat capacity as well as the individual Ising and Potts heat capacities could be computed. In order to calculate the Ising and Potts susceptibilities, the average Ising order parameter as a function of Ising energy, $\left\langle M_{I}\left(E_{I}\right)\right\rangle$, and the average Potts order parameter as a function of Potts energy, $\left\langle M_{P}\left(E_{P}\right)\right\rangle$, were also recorded for each run. Last, the Ising order parameter probability distribution $P\left(M_{I}\right)$, along with the average Ising and Potts energies as functions of the Ising order parameter, $\left\langle E_{I}\left(M_{I}\right)\right\rangle$ and $\left\langle E_{P}\left(M_{I}\right)\right\rangle$, were recorded so that a free energy functional of the Ising order parameter could be calculated.

We present the phase diagrams in the $r, p$ plane where $r$ $=1-e^{-K}, p=w /(1+w)$. The advantage of this particular choice of thermodynamic fields is that each varies between 0 and 1. Each data set obtained at given values of $r$ and $p$ was processed using the histogram method of Ferrenberg and Swendsen ${ }^{9}$ to obtain thermodynamic quantities for a range of nearby values of $p$ at constant $r$. Each transition point was then determined by locating the value of $p$ for which the heat capacity was a maximum. The Ising heat capacity was used for the PP- (percolating paramagnetic) PF (percolating ferromagnetic) transition, the Potts heat capacity was used for the NP- (nonpercolating paramagnetic) PP transition, and the total system heat capacity was used for the NP-PF transition.

These calculated transition points were in good agreement with the known results at $r=1, p=1$, and $r=0$. For instance, although singularities prevented the program from running at $r=1$, the data at $r=0.98$ showed that for $\boldsymbol{L}=40$ the critical values of $p$ for $q=2,3$, and 4 were $0.6694,0.7129$, and 0.7407 , respectively. These compare to $p_{c}=\sqrt{q} /(1+\sqrt{q})$ $=0.6667,0.7101$, and 0.7388 for an infinite system at $r$ $=1$. Due to the negative slope of the phase boundary, $p_{c}$ is expected to be higher at $r=0.98$ than at $r=1$. Estimates of the uncertainties in $p_{c}$ for a given $q, \boldsymbol{L}$, and $r$ were obtained
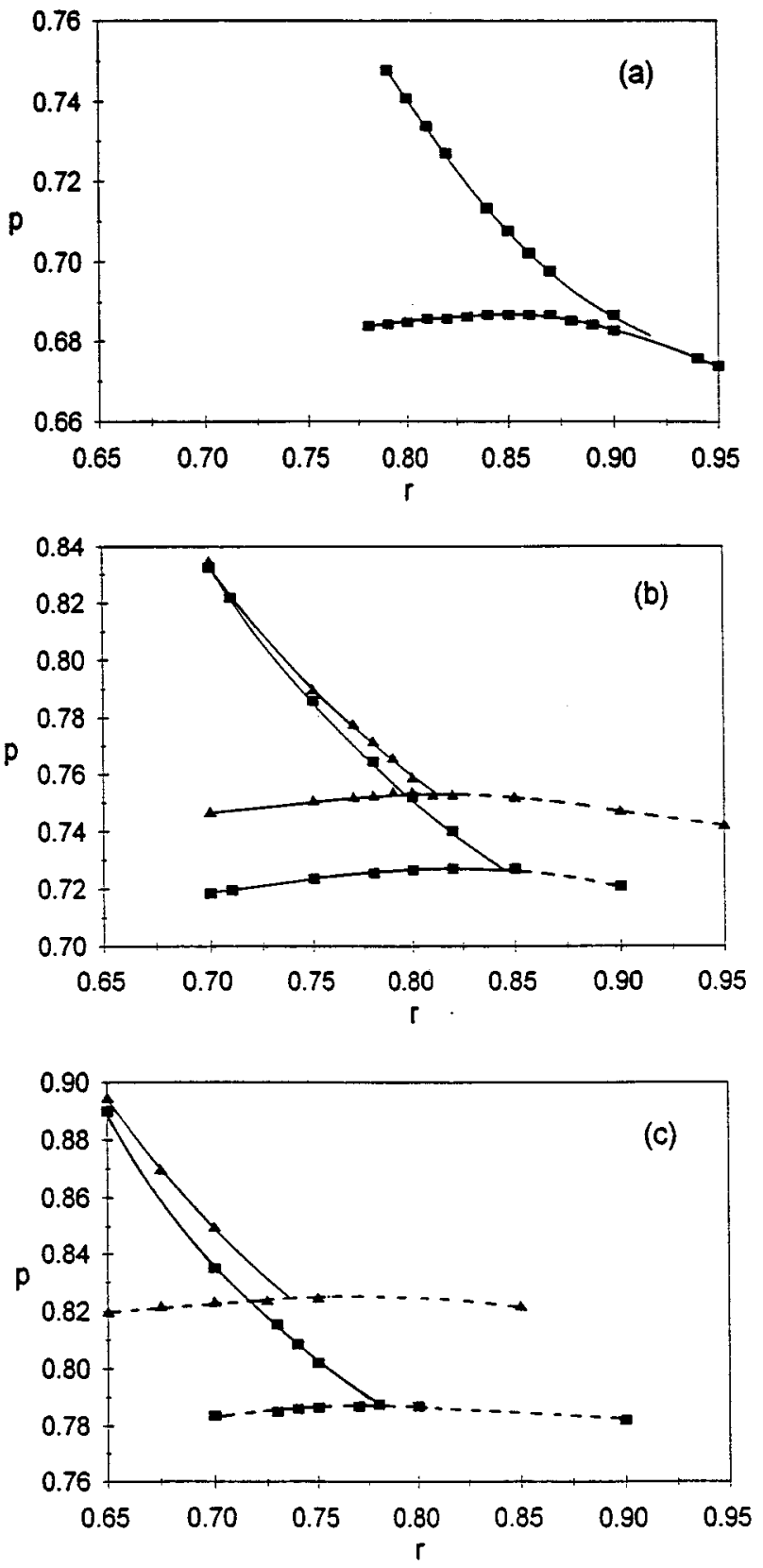

FIG. 1. Phase diagram for (a) $q=2$, (b) $q=3$ (口) and $q=4$ ( $\mathbf{\Delta}$ ), and (c) $q=6(\mathbf{\square})$ and $q=10(\mathbf{\Delta})$. The lines are merely guides to the eye. Solid lines indicate continuous transitions, and dashed lines indicate first-order transitions.

by doing a second Monte Carlo run at or very near the original value of $p$. In all 12 such cases examined, both computed values of $p_{c}$ were within \pm 0.0002 .

It was found that for every value of $q$ and $r$, the critical value of $p$ increased with the lattice size. Extrapolations of graphs of $p_{c}$ vs $1 / \boldsymbol{L}$ indicated that critical values of $p$ for infinite systems would be only about 0.003 higher than those for $\boldsymbol{L}=40$.

The phase diagrams obtained from the simulations are shown in Fig. 1. They are in qualitative agreement with those obtained from the renormalization-group analysis, but the multicritical points occur at significantly lower values of $p$ and $r$. As $q$ decreases, the location of the multicritical point 

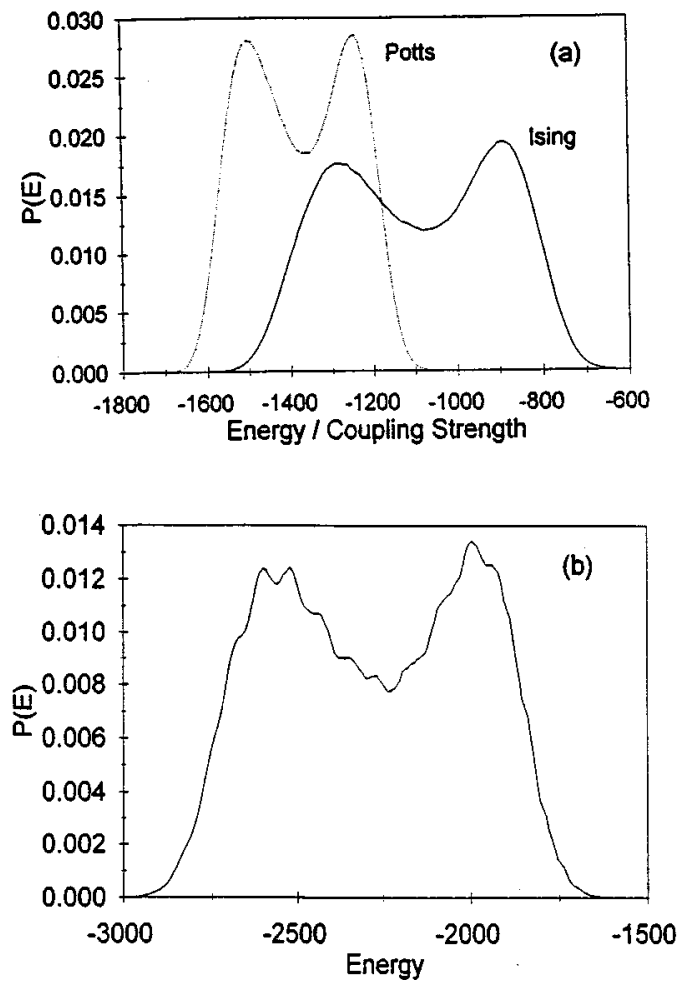

FIG. 2. Energy probability distributions for $q=3$ at $r=0.95$ and $p=0.7161$ for a lattice with $\boldsymbol{L}=30$ : (a) Ising and Potts energies and (b) system energy.

moves to higher values of $r$ and lower values of $p$.

Of special interest to this study is the nature of the phase transitions and how they depend on $q$. The PP-PF transition is an Ising transition and so is continuous for all $q$. The NP-PP transition is a Potts transition and therefore is continuous for $q \leqslant 4$, but first order for $q>4$. The nature of the NP-PF transition was not initially obvious and so was investigated further. For $q>2$ the energy distributions displayed two distinct peaks (see Fig. 2), suggesting a first-order transition. To confirm this the Lee-Kosterlitz function ${ }^{10} F(E)$ was computed for $q=3$ and 4 at $r=0.95$ and 0.98 for $\boldsymbol{L}$ $=14,20,30$, and 40 . Figure 3 shows that $\Delta F(E)$, i.e., the depth of the two minima relative to the height of the barrier separating them, increases with $\boldsymbol{L}$. The effect is much more pronounced for $q=4$, but nonetheless the data show that the transition is first order in both cases.

From Fig. 1(a) it first appeared that for $q=2$ a multicritical point existed for $r<1$; i.e., three phase boundaries seemed to intersect at around $r=0.91$. If true, this would be contrary to the predictions of the renormalization-group analysis which held that the multicritical point was at $r=1$.

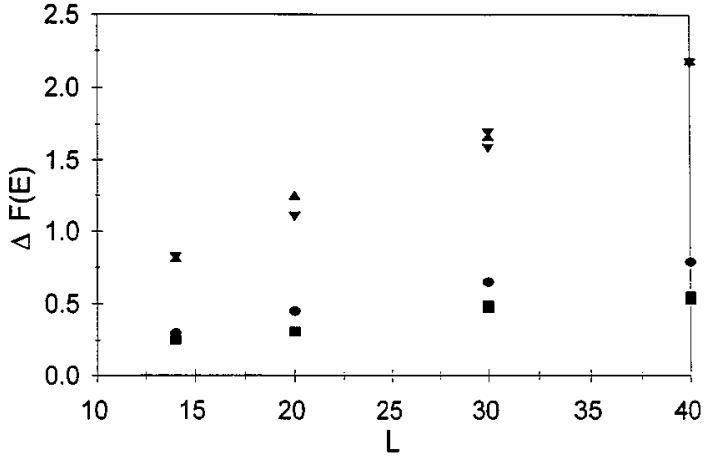

FIG. 3. $\Delta F(E)$ as function of lattice size $\boldsymbol{L}$ for $q=3, r=0.95$ ), $q=3, r=0.98(\boldsymbol{\bullet}), q=4, r=0.95(\boldsymbol{\nabla})$, and $q=4, r=0.98(\boldsymbol{\Delta})$.

To determine whether there existed one phase boundary (NP-PF) or two boundaries (NP-PP and PP-PF) close together, the values of $p_{c}$, as determined by the maxima of the Ising and Potts heat capacities and susceptibilities, were examined in detail. The precision of each $p_{c}$ for an individual run is \pm 0.00005 , although, as mentioned above, the reproducibility from run to run is about \pm 0.0002 . The computed differences of 0.0001 or less for the cases of $q=3$ and 4 indicate that the Ising and Potts systems are ordering together. However, the differences for $q=2$ are significant. They are, at $r=0.95$, for susceptibility $\Delta p_{c}=0.0013$ and for heat capacity $\Delta p_{c}=0.0005$; at $r=0.98$, for susceptibility $\Delta p_{c}=0.0003$ and for heat capacity $\Delta p_{c}=0.0002$. Furthermore, the data show that the Ising spins order at a higher $p$ value than do the Potts spins, consistent with the percolatingparamagnetic to percolating-ferromagnetic boundary being above the nonpercolating-paramagnetic to percolatingparamagnetic boundary. Although the numerical simulations cannot provide absolute proof that the multicritical point does not occur until $r=1$, the data are consistent with the picture of two phase boundaries tangentially approaching each other as $r \rightarrow 1$ or $T \rightarrow 0$.

\section{CONCLUSIONS}

We have established the phase diagram of the square lattice Ising model on percolating clusters by using Monte Carlo simulations. The simulations were performed on the equivalent Ising-Potts model. The Monte Carlo results are consistent with the renormalization-group result ${ }^{1}$ that the nonpercolating-paramagnetic to percolating-ferromagnetic phase transition for any $q>2$ is in the universality class of the $2 q$-state Potts model. Particularly interesting is the occurrence of bicritical points for $2<q \leqslant 4$ and of critical end points for $q>4$. Further studies of critical exponents and amplitudes associated with these multicritical points are needed.
${ }^{1}$ M. Kaufman and J. E. Touma, Phys. Rev. B 49, 9583 (1994).

${ }^{2}$ A. Falicov and A. N. Berker, Phys. Rev. Lett. 74, 426 (1995).

${ }^{3}$ M. Kaufman and D. Andelman, Phys. Rev. B 29, 4010 (1984).

${ }^{4}$ M. E. Fisher and D. R. Nelson, Phys. Rev. Lett. 32, 1350 (1974);
D. R. Nelson, J. M. Kosterlitz, and M. E. Fisher, Phys. Rev. Lett. 33, 813 (1974).

${ }^{5}$ R. R. Netz and A. N. Berker, Phys. Rev. B 47, 15019 (1993).

${ }^{6}$ M. E. Fisher and P. J. Upton, Phys. Rev. Lett. 65, 2402 (1990); 
M. E. Fisher and M. C. Barbosa, Phys. Rev. B 43, 11177 (1991); M. C. Barbosa and M. E. Fisher, ibid. 43, 10635 (1991). ${ }^{7}$ B. Nienhuis, A. N. Berker, E. K. Riedel, and M. Schick, Phys. Rev. Lett. 43, 737 (1979).
${ }^{8}$ F. Y. Wu, Rev. Mod. Phys. 54, 235 (1982).

${ }^{9}$ A. M. Ferrenberg and R. H. Swendsen, Phys. Rev. Lett. 61, 2635 (1988).

${ }^{10}$ J. Lee and J. M. Kosterlitz, Phys. Rev. Lett. 65, 137 (1990). 